\title{
Nuclease-resistant synthetic drug-DNA adducts: programmable drug-DNA conjugation for targeted anticancer drug delivery
}

\author{
Guizhi Zhu ${ }^{1,2}$, Sena Cansiz ${ }^{2}$, Mingxu You ${ }^{1,2}$, Liping Qiu, ${ }^{1,2}$, Da $\mathrm{Han}^{2}$, Liqin Zhang ${ }^{2}$, Lei Mei ${ }^{1}$, Ting Fu ${ }^{1}$, \\ Zhuo Chen ${ }^{1}$ and Weihong Tan ${ }^{1,2}$
}

Targeted drug delivery is poised to improve cancer therapy, for which synthetic DNA can serve as targeting ligands (for example, aptamers) or drug nanocarriers. Inspired by natural DNA adducts, we report synthetic drug-DNA adducts (DDAs) for targeted anticancer drug delivery. Multiple copies of anthracycline drugs were site specifically (on deoxyguanosine) conjugated on each DNA, enabling programmable design of DNA and drugs for DDA preparation. DDAs were nuclease-resistant and stable for storage, yet gradually released drugs at physiological temperature. DDAs maintained DNA functionalities, including hybridizationmediated DNA nanoadduct formation and aptamer-mediated target recognition and targeted drug delivery into cancer cells. In a tumor xenograft mouse model, doxorubicin-aptamer adducts significantly inhibited target tumor growth while reducing the side effects. Using histopathological analysis and in situ immunohistochemical analysis of caspase-3 cleavage in mouse tumor and heart, DDAs were confirmed to have a potent antitumor efficacy while reducing tissue deformation and apoptosis in the heart, thus providing a new therapeutic avenue to prevent cardiomyopathy, the most dangerous side effect of doxorubicin leading to heart failure. Overall, DDAs are promising for scale-up production and clinical application in targeted anticancer drug delivery. NPG Asia Materials (2015) 7, e169; doi:10.1038/am.2015.19; published online 27 March 2015

\section{INTRODUCTION}

Conventional chemotherapeutic drugs lack the specificity to localize cancer cells, and hence systemic drug distribution leads to toxicity in healthy tissue and organs, ${ }^{1}$ which makes it difficult to escalate drug dose to enhance the therapeutic efficacy. For example, doxorubicin (Dox), a frequently used cancer chemotherapeutic drug, can cause cardiomyopathy (incidence: $30 \%),{ }^{2}$ which often leads to congestive heart failure, resulting in $\sim 50 \%$ mortality. $^{2}$ In contrast, targeted therapy seeks to deliver drugs specifically to cancer cells. ${ }^{3}$ Active targeting achieves this by using molecular targeting elements, including antibodies, aptamers, growth factors and vitamins, ${ }^{4,5}$ which can specifically bind to overexpressed cognate receptors on target cancer cells. ${ }^{6}$ Indeed, antibody-drug conjugates are of intensive interest to the pharmaceutical industry. However, a simple and cost-effective antibody-drug conjugate technology to site specifically conjugate multiple copies of drugs with one antibody is still a daunting challenge. Passive targeting exploits the leaky blood vasculature and poor lymphatic drainage in tumors that allow nanocarriers, such as polymer nanoparticles, ${ }^{3}$ liposomes, ${ }^{7}$ gold nanoparticles ${ }^{8}$ and nucleic acid nanostructures, ${ }^{9-17}$ to access and accumulate in the tumor by the enhanced permeability and retention effect. ${ }^{3}$

DNA, the genetic carrier in nature, has been exploited as both synthetic targeting elements (for example, aptamers, CpG DNA), ${ }^{18-21}$ and nanocarriers ${ }^{9-17}$ for both active and passive targeted drug delivery, owing to such features as ease of reproducible synthesis and modification, biodegradability, sequence programmability, structure predictability of the resultant DNA drug carriers and intrinsic targeting or therapeutic functionalities. DNA aptamers, screened by Systematic Evolution of Ligands by EXponential enrichment, ${ }^{22,23}$ are excellent candidates as targeting elements. ${ }^{24}$ Recently, our group developed cellSystematic Evolution of Ligands by EXponential enrichment to select aptamers for living cells of various cancers. ${ }^{25-28}$ DNA aptamers possess many remarkable features, such as facile screening against various targets, automated synthesis and modification, strong binding affinity

${ }^{1}$ Molecular Science and Biomedicine Laboratory, State Key Laboratory of Chemo/Bio-Sensing and Chemometrics, College of Chemistry and Chemical Engineering and College of Biology, Collaborative Innovation Center for Molecular Engineering and Theranostics, Hunan University, Changsha, China and ${ }^{2}$ Departments of Chemistry, Physiology and Functional Genomics, Center for Research at the Bio/Nano Interface, Shands Cancer Center, UF Genetics Institute and McKnight Brain Institute, University of Florida, Gainesville, FL, USA

Correspondence: Professor W Tan, Molecular Science and Biomedicine Laboratory, State Key Laboratory of Chemo/Bio-Sensing and Chemometrics, College of Chemistry and Chemical Engineering and College of Biology, Collaborative Innovation Center for Molecular Engineering and Theranostics, Hunan University, Changsha, 410082, China and Departments of Chemistry, Physiology and Functional Genomics, Center for Research at the Bio/Nano Interface, Shands Cancer Center, UF Genetics Institute and McKnight Brain Institute, University of Florida, Gainesville, FL 32611-7200, USA.

E-mail: tan@chem.ufl.edu

or Professor Z Chen, Molecular Science and Biomedicine Laboratory, State Key Laboratory of Chemo/Bio-Sensing and Chemometrics, College of Chemistry and Chemical Engineering and College of Biology, Collaborative Innovation Center for Molecular Engineering and Theranostics, Hunan University, Changsha 410082, China.

E-mail: zhuochen@hnu.edu.cn

Received 8 July 2014; revised 14 December 2014; accepted 23 December 2014 
and low cytotoxicity or immunogenicity. ${ }^{24}$ Because of the sequence programmability, DNA has been engineered into various drug nanocarriers. ${ }^{10,12-14}$ Moreover, DNA per se can serve as therapeutics, such as DNA antisense, ${ }^{29}$ aptamers ${ }^{24}$ and immunostimulatory CpG motifs, ${ }^{21}$ allowing combination therapy in DNA-based drug delivery systems.

These remarkable features have allowed DNA to be developed into targeted drug delivery systems, in which drugs are conjugated with DNA both noncovalently ${ }^{15,17-19}$ (for example, intercalation of anthracycline drugs into double-stranded DNA-CG sites ${ }^{15,18,19}$ and photosensitizer TMPyp4 into G-quadruplexes) and covalently. ${ }^{30,31}$ However, noncovalent conjugation typically requires specific DNA sequences or modification with additional sequences for drug conjugation; yet current solid-phase DNA synthesis technology can only synthesize DNA of limited lengths. Likewise, covalent drug-DNA conjugation typically relies on DNA modification with reactive groups, and complicated organic synthesis with low yield. ${ }^{30,31}$ Moreover, when systemically administered in a physiological environment, DNA complexes could be degraded or dissociated by ubiquitous nucleases, dilution to low concentrations by blood and strong shear forces. ${ }^{32}$ To prevent nuclease degradation, current strategies again rely on either chemical modification (for example, phosphorothioate linkages and 2 '-O-methyl ${ }^{32}$ or the use of other nanomaterials. ${ }^{33}$

Addressing these shortcomings calls for a simple, yet a versatile and programmable platform for the development of a new chemical species, or adduct, enabling site-specific covalent conjugation of drugs and unmodified DNA, such that the resultant product, that is, drugDNA adducts (DDA), is additionally endowed with resistance to nuclease cleavage. In nature, adducts can be formed between the genomic DNA (dsDNA) and drugs such as Dox and cisplatin. ${ }^{34-36}$ Indeed, formation of adducts between genomic DNA and drugs, or hazardous chemicals, is one of the mechanisms of their genotoxicity. ${ }^{37}$ Inspired by this, we developed a simple, yet versatile and programmable platform to make synthetic DDAs in vitro by using anticancer drugs and DNA (aptamers or DNA building blocks) for targeted cancer therapy. The preparation of DDAs is simple and avoids complicated organic synthesis. DDA formation exploits the reactive $2-\mathrm{NH}_{2}$ on deoxyguanosine, avoiding any DNA modification with reactive groups. The site-specific drug conjugation on deoxyguanosine enables programmable drug conjugation, with typically multiple drug copies conjugated on one DNA. DDAs can be formed by using various anthracycline drugs and essentially any deoxyguanosine-containing DNA. DDAs are strongly resistant to nuclease degradation. Furthermore, they maintain the functionalities of DNA backbones, including (1) hybridization-mediated drug nanocarrier formation and (2) aptamer-mediated cancer cell recognition and selective drug delivery into target cancer cells. In a tumor xenograft mouse model, Dox-aptamer adduct demonstrated potent antitumor efficacy and reduced side effects. By histopathological analysis and immunohistochemical analysis of cleaved caspase-3, a marker for apoptosis, ${ }^{38}$ we confirmed that drug-aptamer adducts (DAAs) have potent toxicity in tumors and reduced toxicity in the heart, providing a new way to prevent cardiomyopathy, a lethal side effect of Dox. Taken together, these results suggest that DDAs are promising for programmable applications in targeted drug delivery as well as scale-up production.

\section{EXPERIMENTAL PROCEDURE DDA preparation}

By using a modified protocol, ${ }^{36}$ DDAs were prepared by incubating drugs $(500 \mu \mathrm{M})$, DNA $(20 \mu \mathrm{M})$ and formaldehyde $(0.37 \%)$ in reaction buffer $(20-\mathrm{mm}$ sodium phosphate, 150 -mm $\mathrm{NaCl}$ and 0.5-mм EDTA; $\mathrm{pH} 7.0$ ) at $10^{\circ} \mathrm{C}$ for $10 \mathrm{~h}$.
The resultant DDAs were purified by reversed phase high-performance liquid chromatography (HPLC) (ProStar, Varian, Walnut Creek, CA, USA) on a C-18 column using 0.1-M triethylamine acetate (TEAA, Glen Research Corp., Sterling, VA, USA) and acetonitrile (Sigma Aldrich, St Louis, MO, USA) as the eluent. Samples were concentrated using a freeze dryer (Thermo Electron Co., Waltham, MA, USA), subjected to desalting or buffer exchange by using dulbecco's phosphate-buffered saline (DPBS) buffer (Sigma Aldrich), and stored at $-20^{\circ} \mathrm{C}$ for future use.

\section{DDA determination and quantification}

DDA formation was verified and quantified by agarose gel electrophoresis (3\%, $100 \mathrm{~V}, 50 \mathrm{~min}, 4^{\circ} \mathrm{C}$ ), electrospray ionization mass spectrometry (ESI-MS) (Novatia, Inc., Newtown, PA, USA) and UV-Vis spectrometry by using a Cary Bio-100 UV-Vis spectrometer (Varian). Molar extinction coefficients of 11 $500 \mathrm{M}^{-1} \mathrm{~cm}^{-1}$ at $480 \mathrm{~nm}$ for free Dox and $7677 \mathrm{M}^{-1} \mathrm{~cm}^{-1}$ at $506 \mathrm{~nm}$ for covalently bound Dox in DDAs were used for adduct quantification by UV-Vis spectrometry, as described in a previous report. ${ }^{36}$

\section{Resistance of DDAs to nuclease degradation}

DNA $(2 \mu \mathrm{M})$ or DDAs (4- $\mu \mathrm{M}$ DNA equivalents) were prepared in DNase I reaction buffer (10-mM Tris- $\mathrm{HCl}, 2.5-\mathrm{mM}^{\mathrm{MgCl}_{2}}, 0.5-\mathrm{mm} \mathrm{CaCl}_{2}, \mathrm{pH} 7.6$ at $\left.25^{\circ} \mathrm{C}\right)$. DNase $\mathrm{I}\left(0.05 \mathrm{U} \mathrm{\mu l}^{-1}\right)$ was added to the resultant solutions, followed by incubation for $10 \mathrm{~min}$ at room temperature and deactivation by heating at $75^{\circ} \mathrm{C}$ for $5 \mathrm{~min}$ prior to agarose gel electrophoresis analysis. To study the enzymatic activity of DNase I premixed with DDAs, either DNA $(4 \mu \mathrm{M})$ or DDAs (8- $\mu \mathrm{M}$ DNA equivalents) were again prepared in DNase I reaction buffer

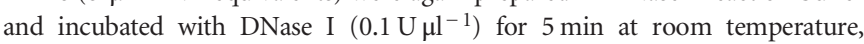
followed by the addition of $\mathrm{DNA}^{\prime}(4 \mu \mathrm{M})$ and further incubation for $5 \mathrm{~min}$ at room temperature. The resultant samples were deactivated by heating at $75^{\circ} \mathrm{C}$ for $5 \mathrm{~min}$ and then subjected to agarose gel electrophoresis $(3 \%, 100 \mathrm{~V}, 50 \mathrm{~min}$, $\left.4^{\circ} \mathrm{C}\right)$.

\section{Drug release in buffer solution}

Drug release in solution was monitored by HPLC (ProStar, Varian) on a C-18 column, by using 0.1-M triethylamine acetate (TEAA, Glen Research Corp.) and acetonitrile (Sigma Aldrich) as the eluent, to determine the fraction of drug remaining bound to the DNA. DDAs (50- $\mu$ M Dox equivalents, $100 \mu \mathrm{l})$ in DPBS were incubated at 4 or $37^{\circ} \mathrm{C}$ and analyzed by HPLC at various time points. The fraction of Dox remaining bound to the DNA was determined at each time point by the ratio of the integrated area (measured by using ImageJ software (National Institutes of Health, Bethesda, MD, USA)) under the peak corresponding to remaining DDAs to the area under the peak corresponding to free Dox at an absorbance of $490 \mathrm{~nm}$. The resultant fraction was then converted to the fraction of released drug, $F_{\text {released. }}$. Data points were fit by nonlinear regression using the Origin 8 software (Miami, FL, USA) to a firstorder release model:

$$
F_{\text {released }}=\alpha\left[1-\exp \left(-\ln (2) t / t_{1 / 2}\right)\right]
$$

( $t$ is the time (h) of incubation, $t_{1 / 2}$ is the half-life (h) of drug release and a (\%) is the maximum fraction of drug release).

\section{In vitro cytotoxicity assay}

The cytotoxicity of free drugs or DAAs was evaluated using CellTiter 96 cell proliferation assay (Promega, Madison, WI, USA). Cells $\left(5 \times 10^{4}\right.$ cells per well) were treated with free Dox and DAAs in medium without fetal bovine serum (FBS) $\left(37^{\circ} \mathrm{C}, 5 \% \mathrm{CO}_{2}\right)$. After incubation for $1 \mathrm{~h}$, cells were precipitated by centrifugation, followed by removal of $85 \%$ supernatant medium and addition of fresh medium (10\% FBS, $200 \mu \mathrm{l})$ for further cell growth $(48 \mathrm{~h})$. Then cells were again precipitated, and medium was removed. CellTiter reagent $(20 \mu \mathrm{l})$ diluted in fresh medium (10\% FBS, $100 \mu \mathrm{l})$ was added to each well and incubated for $1-2 \mathrm{~h}$. The absorbance $(490 \mathrm{~nm})$ was recorded by using a microplate reader (Tecan Safire microplate reader, San Jose, CA, USA). Cell viability was determined according to the manufacturer's description. 


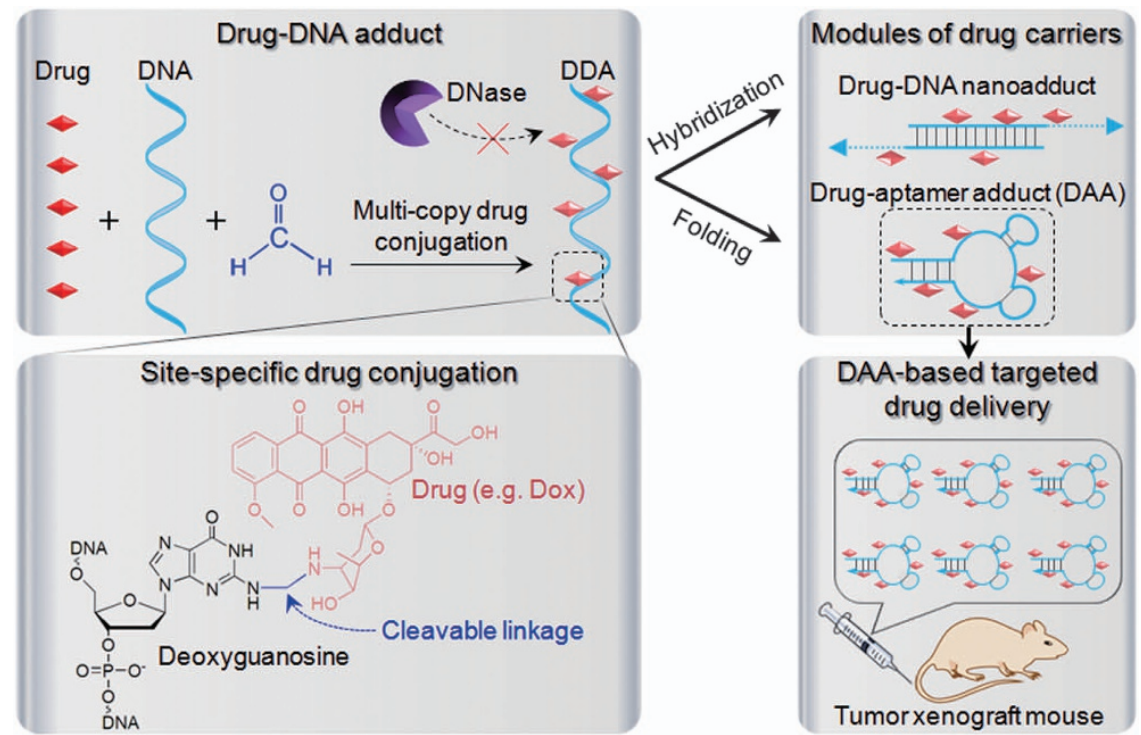

Figure 1 Schematic illustration of nuclease-resistant synthetic drug-DNA adducts as a simple, yet versatile and programmable platform for targeted anticancer drug delivery. In a simple reaction, multiple copies of drugs (e.g., doxorubicin) were specifically conjugated on deoxyguanosine of DNA, making it programmable to design DNA and drugs for drug-DNA adduct-based drug delivery. Drug fluorescence was quenched upon conjugation to DNA. Drug-DNA adducts were stable at relatively low temperature, yet the cleavable methylene linkage (heat labile) between drug moieties and DNA allows for drug release at a physiological temperature. Drug-DNA adducts were resistant to nuclease degradation and maintained the functionalities of specific cancer cell recognition by drug-aptamer adducts and hybridization-mediated formation of drug-DNA nanoadducts. Drug-aptamer adducts were further studied for targeted anticancer drug delivery in a xenograft tumor mouse model, and showed potent antitumor efficacy while significantly reducing the side effects of doxorubicin.

\section{In vivo evaluation of DAAs for targeted cancer therapy}

NOD. Cg-Prkdc (scid) IL2 mice were purchased from Jackson Laboratory (Bar Harbor, ME, USA). The animal use protocol was approved by the University of Florida Institutional Animal Care and Use Committee. The tumor xenograft mouse model was developed by subcutaneously injecting $8 \times 10^{6}$ in vitropropagated CEM cells (in 100- $\mu$ l DPBS buffer) into Cg-Prkdc (scid) IL2 mice on the back. Dorsal tumor nodules were allowed to grow to a volume of $\sim 100 \mathrm{~mm}^{3}$ before treatment initiation. Tumor-bearing mice were randomly assigned to four groups, with five mice in each group treated with (i) $\mathrm{sgc}$, (ii) free Dox, (iii) control DNA-Dox adduct and (iv) sgc8-Dox adduct. Dox dosage was kept at $3 \mathrm{mg} \mathrm{kg}^{-1}$, and the sgc8 dosage in group (i) was the same as that in group (iv). Every other day, drugs were injected through tail veins, mice were weighted to assess side effects and tumor sizes for each mouse were measured with calipers. Tumor volume was calculated by using:

$$
\text { Tumor volume }=\left(\text { length } * \text { width }^{2}\right) / 2
$$

Mice were killed when tumor length or width exceeded $1.5 \mathrm{~cm}$, or when ulceration or morbidity developed. Data were analyzed by using GraphPad Prism 5 software (GraphPad Software, San Diego, CA, USA). The heart, kidney and tumor of each mouse were collected after mice were killed and fixed in $10 \%$ formalin for $24 \mathrm{~h}$ at room temperature. Tissues were then embedded in paraffin for sectioning. Hematoxylin and eosin staining and in situ immunohistochemical analysis of cleaved caspase-3, by using cleaved caspase-3 (Asp175) antibody (Cell Signaling Technology, Inc., Danvers, MA, USA), were performed in the Molecular Pathology Core Laboratory at the University of Florida.

\section{RESULTS AND DISCUSSION}

\section{DDA preparation, determination and characterization}

As a model, a DNA aptamer $\operatorname{sgc} 8^{26}$ (sequence in Supplementary Table S1), Dox and formaldehyde (a reducing and crosslinking agent for protein or nucleic acid crosslinking) were used to prepare synthetic DDA (Figure 1). Sgc8, which can specifically recognize many types of cancer cells, ${ }^{26,39}$ was used for the long-term goal of targeted drug delivery. DDA was prepared by incubating DNA, excess drug and formaldehyde in reaction buffer at $10^{\circ} \mathrm{C}$ overnight, following purification by HPLC to remove any residual drug and formaldehyde. As shown in Supplementary Figure S1A, DDA in HPLC displayed a strong absorbance at $490 \mathrm{~nm}$ from the drug only and $260 \mathrm{~nm}$ from both the drug and DNA, whereas free DNA, or reaction solution without formaldehyde, displayed no apparent absorbance at $490 \mathrm{~nm}$. Purified DDA was lyophilized, desalted and stored at $-20^{\circ} \mathrm{C}$.

The formation of DDA was verified by: (1) characteristic absorbance in HPLC and UV-Vis spectrometry (Supplementary Figure S3A, Figure 2c); (2) mobility shift in gel electrophoresis compared with free DNA (Supplementary Figure S2); and (3) ESI-MS, in which the DNA mass peaks shifted from $14592.8 \mathrm{Da}$ to a series of larger molecular weights (Figures 2a and $\mathrm{b}$ ). The mass differences between DDAs and pure DNA correspond to molecular weights of about 1-6 copies of drug and methylene linker (Supplementary Table S2), indicating conjugation of multiple copies of drug on one DNA. Based on the characteristic drug absorbance, an average of $5.1 \pm 0.3$ (s.d., $n=3$ ) drug molecules were determined to be conjugated per DNA in DDAs. The lower average number of drug molecules ( 3.21. Figure 2b) attached on one DNA observed in ESI-MS compared with UV-Vis was presumably caused by the degradation of DDAs (to be explained later) prior to ESI-MS analysis. We also observed a red-shift in DDA absorbance compared with free drugs (Supplementary Figure S3B) in UV-Vis spectrometric analysis and dramatic drug fluorescence quenching in DDAs (Figure 2d, Supplementary Figure S3C), providing simple alternative ways to determine DDA formation.

For efficacious drug delivery, it is essential for drug conjugates to be stable for storage and release drugs at target disease sites. ${ }^{37}$ Previous studies have shown that adducts formed from genomic DNA and anthracycline drugs are heat labile. ${ }^{34}$ This implies that DDAs would be stable at relatively low temperature, yet unstable and able to release drugs at relatively high temperature. We first investigated the stability of DDAs at $4{ }^{\circ} \mathrm{C}$ in physiological buffer solution. A series of DDA aliquots was incubated at $4{ }^{\circ} \mathrm{C}$ for different time lengths, followed by 
a

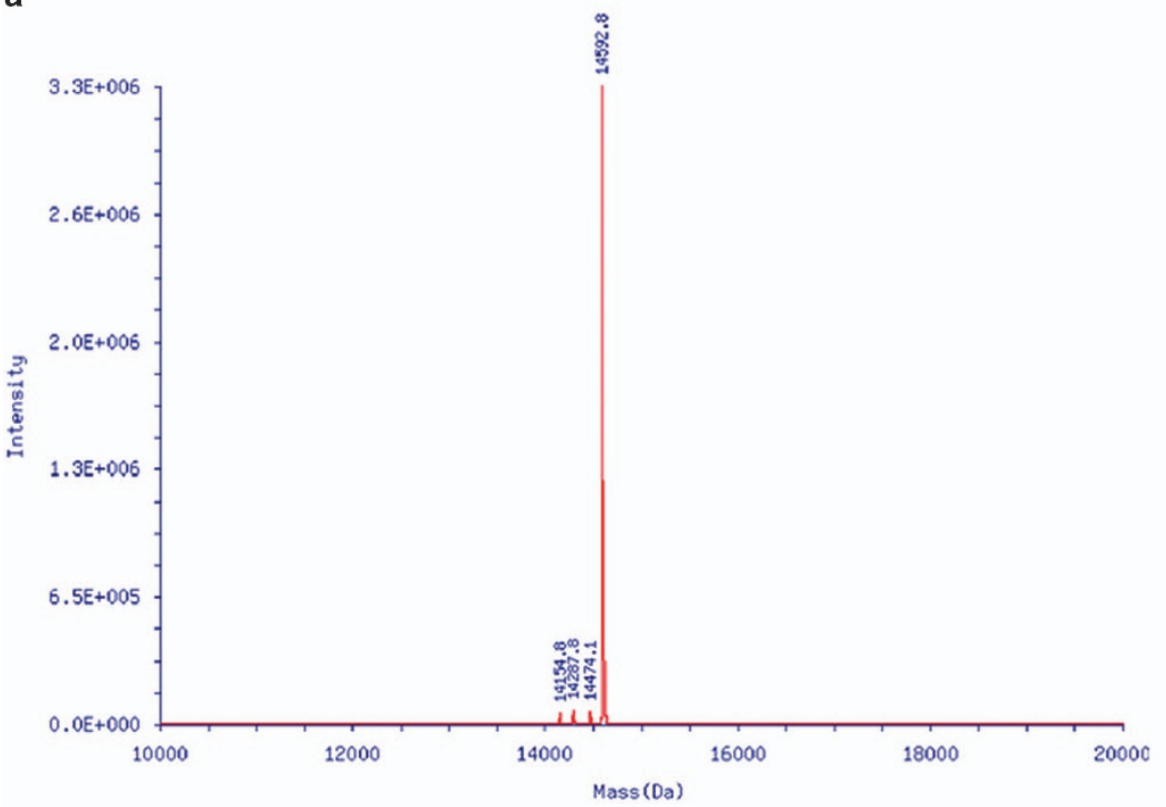

b

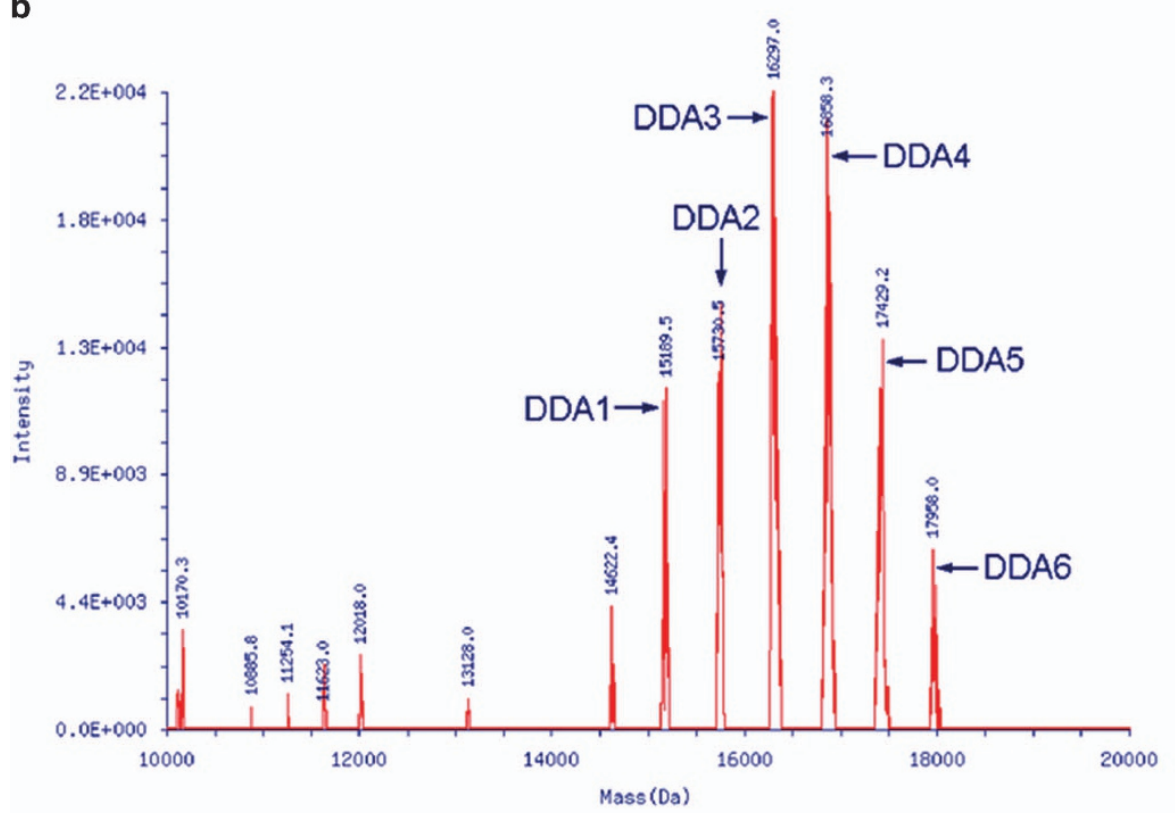

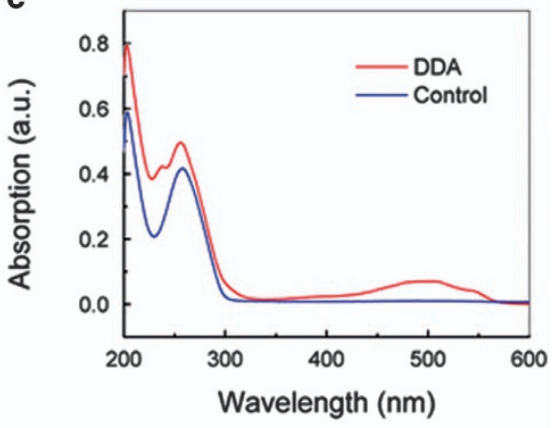
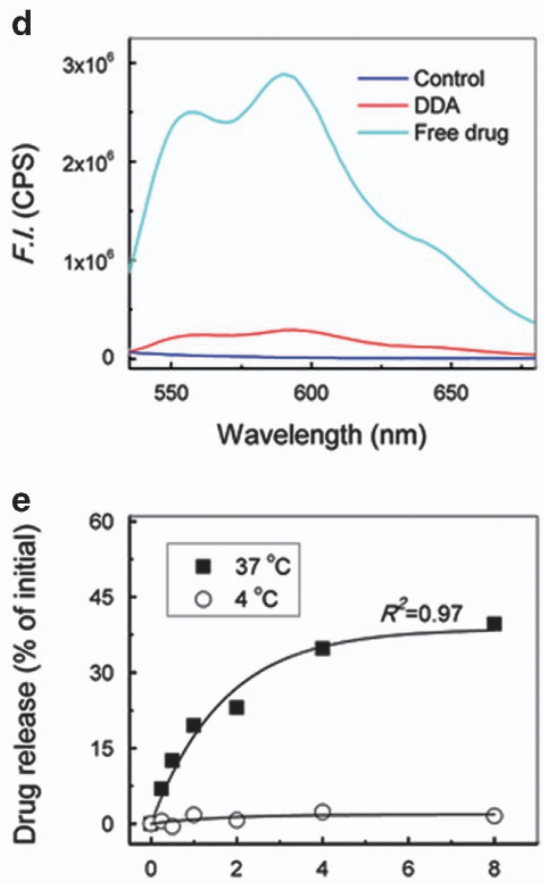

Time (h)

$\mathbf{f}$

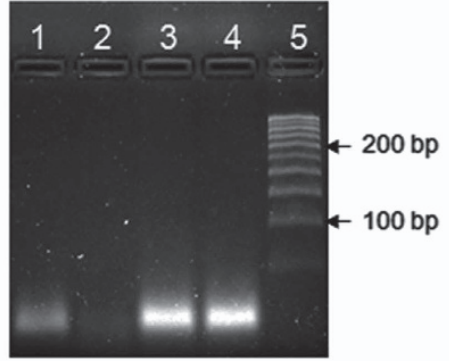

Figure 2 Characterization of drug-DNA adducts. (a and $\mathbf{b}$ ) Deconvoluted electrospray ionization mass spectrometry spectra of DNA (sgc8-5T-biotin) (a) and its drug-DNA adducts with doxorubicin (b). Compared with DNA, the peaks (drug-DNA adducts 1-6) shifted to larger molecular weights in drug-DNA adducts (b), corresponding to drug-DNA adducts with 1-6 copies of doxorubicin, respectively, conjugated on one DNA (Supplementary Table S2). (5T: a spacer; Biotin: for streptavidin conjugation in following study) (c) UV-Vis absorption spectra of drug-DNA adducts and control (reaction solution without formaldehyde). (d) Fluorescence spectra of free Dox $(2 \mu \mathrm{m})$, drug-DNA adducts (2- $\mu \mathrm{m}$ doxorubicin equivalents) and control. (e) High stability of drug-DNA adducts at $4{ }^{\circ} \mathrm{C}$ and conditional drug release at a physiological temperature $\left(37^{\circ} \mathrm{C}\right)$. (Solid lines: fit curves) (f) An agarose gel electrophoresis image

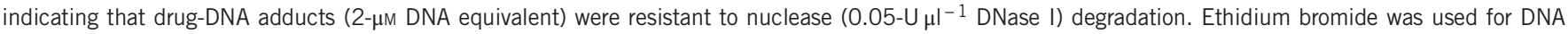
staining. The weaker intensity in lane 1 compared with that in lanes 3 or 4 is likely because of the energy transfer from ethidium bromide to conjugated doxorubicin in drug-DNA adducts. (lane legends: 1, DNA; 2, DNA+DNase I; 3, drug-DNA adducts; 4, drug-DNA adducts+DNase I; and 5, DNA marker).

determination of the amount of liberated drugs by using HPLC. No significant amount of free drug was detected over $8 \mathrm{~h}$, indicating high stability of DDAs at $4{ }^{\circ} \mathrm{C}$ (Figure 2e). Then, the kinetics of drug release from DDAs was studied at a physiological temperature. Similarly, a series of DDA aliquots were incubated at $37^{\circ} \mathrm{C}$ for different time lengths, and drug release was monitored by HPLC. The first-order half-life of drug release was determined to be $1.16 \mathrm{~h}$, with the maximum release of $38.4 \%$ of the initial drug amount under these 
a

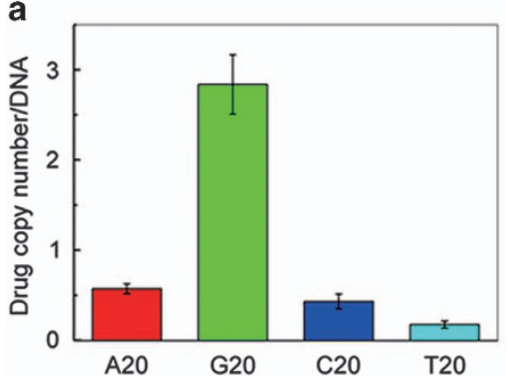

b

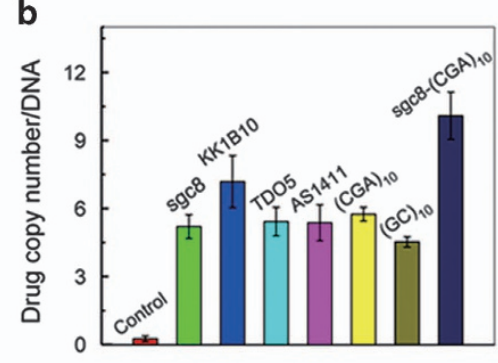

c

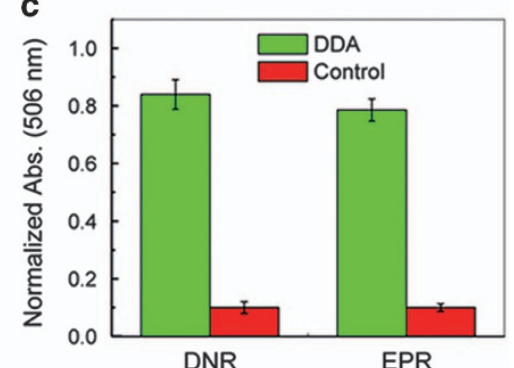

Figure 3 Site-specific and programmable drug-DNA adduct formation. (a) Average drug copy numbers per polynucleotide in drug-DNA adduct prepared from doxorubicin and polynucleotides (A20, G20, C20 and T20), indicating that deoxyguanine had the highest reactivity for drug-DNA adduct formation. This provides a guideline to programmably design DNA and drugs for drug-DNA adduct-based drug delivery systems. (b) Drug-DNA adduct formation with doxorubicin and various DNA sequences. (c) Drug-DNA adduct formation using DNA (sgc8-5 T) with commonly used daunorubicin and enhanced permeability and retention, respectively. Purified drug-DNA adducts were quantified based on absorbance at $506 \mathrm{~nm}$, which was normalized to that of the corresponding control. (Control: reaction using sgc8-5T without formaldehyde).

conditions (Figure 2e). Incomplete drug release is likely because the reaction reached equilibrium under the experimental conditions. Overall, these results indicate that DDAs are exceptionally stable for storage at a relatively low temperature $\left(4^{\circ} \mathrm{C}\right)$, but drugs are released at an appreciable rate at a physiological temperature, providing the basis for efficacious DDA-based targeted drug delivery.

Another remarkable feature of DDAs is the exceptional resistance to nuclease degradation. In our study, DDAs and the DNA counterpart were treated with DNase I and analyzed by using gel electrophoresis. Although DNA was rapidly degraded, DDAs were much less susceptible to enzymatic cleavage than their DNA counterpart (Figure 2f). To rule out the possibility that the decreased cleavage resulted from inhibition of the enzymatic activity of DNase I by DDAs, DNase I was premixed with DNA and DDAs, respectively, followed by introduction of a longer DNA, termed as DNA'. DNA' was degraded in both mixtures, ruling out the possibility that the activity of DNase I premixed with DDAs was inhibited (Supplementary Figure S4). Although the mechanism underlying nuclease resistance is still unknown, it presumably involves conformational constraint resulting from drug conjugation, which prevents binding of DDAs with nucleases for enzymatic cleavage. Without any other chemical modification or conjugation with nanomaterials, DDAs provide a simple and novel approach to protect DNA in drug delivery.

\section{Site-specific drug-DNA conjugation in programmable DDA formation}

To elucidate the drug conjugation sites on DNA, we studied the ability of different deoxynucleosides to form DDAs. In adducts formed between drugs and dsDNA, previous studies showed that the methylene group generated from formaldehyde linked the $3-\mathrm{NH}_{2}$ group of Dox on one side to the $2-\mathrm{NH}_{2}$ of guanine on the other side. ${ }^{35}$ In our preliminary studies, 20 -mer polyA, polyG, polyC and polyT were then, respectively, used in the reaction for DDA formation. Results showed that polyG was most reactive to adduct formation, while polyA, polyC and polyT had a very-low to negligible reactivity, despite the presence of $\mathrm{NH}_{2}$ groups in adenosine and cytosine (Figure 3a). This demonstrates the site specificity of DDA formation, and implies that the number of drugs conjugated onto one DNA should be determined mainly by the G content, making it programmable to design DNA for DDA-based drug delivery. This indicates the site specificity of DDA formation at the nucleobase level, and thus the number of drugs conjugated on one DNA is determined mainly by the G content, making it programmable to design DNA for DDA-based drug delivery. Even though the conjugation efficiency is not as high as some other reactions, such as click chemistry, ${ }^{4}$ the use of intact DNA without any other modification makes this preparation extremely simple and cost-effective, which is promising for future production scale-up. In addition, adduct formation primarily on deoxyguanosine is speculated to cause drug fluorescence quenching in DDAs, given that deoxyguanosine can quench fluorescence of some proximal fluorophores through electron transfer. ${ }^{40}$ Furthermore, DDAs were formed using a variety of DNA strands, including aptamers (KK1B10, TD05), ${ }^{27,28}$ modified aptamers ( $\operatorname{sgc} 8$-(CGA) $)_{10}$ ) and nonaptamer DNA $\left((\mathrm{CGA})_{10},(\mathrm{GC})_{10}\right)$ with diverse nucleotide compositions or sequences (Figure 3b). Even though Dox intercalated in dsDNA, the physically intercalated Dox in sgc8-(CGA) ${ }_{10}$, which forms a homodimer containing dsDNA, was removed during HPLC purification (Supplementary Figure S1B).

In addition to programming DNA for DDA formation, Dox used in DDAs can also be programmably replaced with other drugs. Many anthracycline drugs have structures analogous to Dox, including the commonly used daunorubicin and epirubicin (enhanced permeability and retention) in cancer therapy. As examples, commonly used daunorubicin and enhanced permeability and retention were demonstrated to be able to form DDAs with DNA (Figure 3c). Overall, these results demonstrate the site specificity of DDA formation, making DDA formation programmable to versatile DNA strands and anthracycline drugs.

To explore the applicability of DDAs as building blocks for drug nanocarriers, we then studied whether the DNA backbones in DDAs maintained hybridization ability, which is fundamental to the assembly of many DNA nanocarriers. A bispecific aptameric nanocarrier, assembled by hybridization of the two toeholds extending from aptamers sgc 8 and KK1B10, ${ }^{15}$ was used as a model (Supplementary Figure S5A). The monomers sgc8-hyb and KK1B10-hyb were first used to prepare DDAs with Dox, and the resultant two DDAs were mixed to allow hybridization. Agarose gel electrophoresis clearly demonstrated the formation of a bivalent aptamer structure by using DDA building blocks, verifying that DDAs maintained the ability to form drug-DNA nanoadducts by hybridization of predesigned DDA building blocks (Supplementary Figure S5B).

\section{Drug-aptamer adducts for in vitro targeted drug delivery}

We next evaluated whether the DNA aptamer backbones in DAAs retained the ability to specifically recognize targets. As mentioned above, the DDAs, or DAAs in this case, were prepared intentionally by 
a
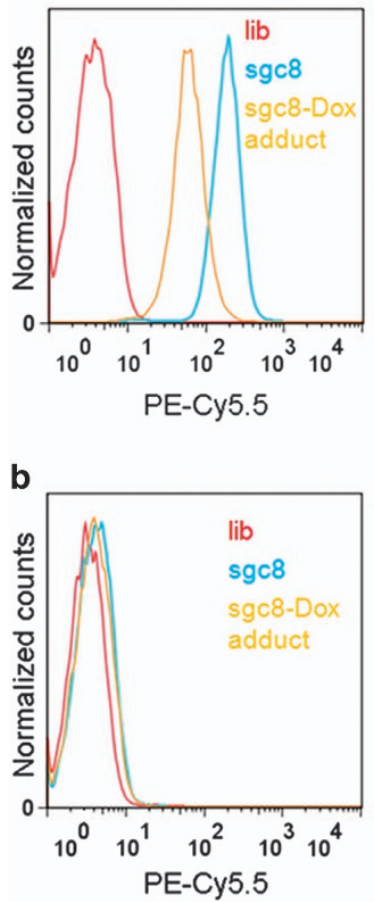

C
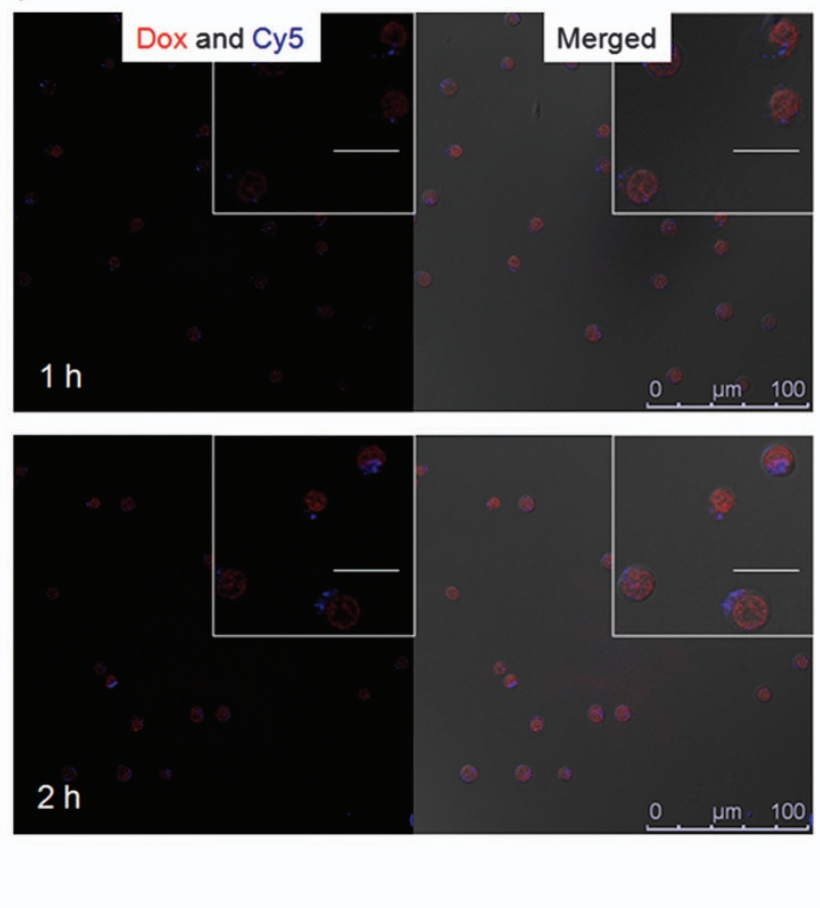

d

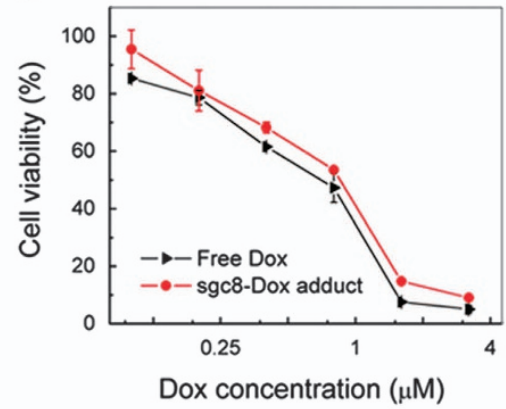

e

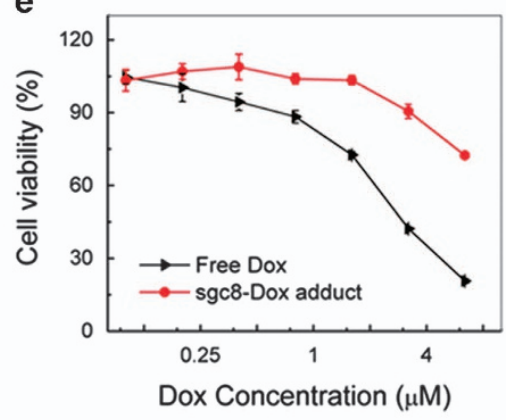

Figure 4 Drug-DNA adducts for targeted anticancer drug delivery in vitro. (a and $\mathbf{b}$ ) Flow cytometry data showing the selective recognition of sgc8-Dox adduct to target CEM cells (a), but not to nontarget Ramos cells (b). (sgc8: Biotinylated; lib: random sequences) (c) confocal laser scanning microscopy images displaying the intracellular behaviors of sgc8-doxorubicin adduct (2- $\mu \mathrm{m}$ doxorubicin equivalents) in CEM cells after treatment for 1 and $2 \mathrm{~h}$, respectively. (sgc8: labeled with Cy5; insets: enlarged cells; inset scale bar: $20 \mu \mathrm{m}$.) (d and e) MTS assay results indicating selective cytotoxicity of sgc8-doxorubicin adduct in CEM cells (d), but much less in Ramos cells (e).

using DNA modified from aptamer sgc8, which can bind to target protein PTK7, a pseudokinase overexpressed on many types of cancer cells, including target CEM cells, but not on nontarget Ramos cells. ${ }^{26,39}$ The binding ability of DAAs prepared from biotinylated sgc8-5 T was monitored by using a streptavidin-PE-Cy5.5 dye conjugate in flow cytometry. The dramatic enhancement of fluorescence intensity of target CEM cells incubated with this DAA compared with the negative control suggests its selective binding ability to CEM cells (Figure $4 \mathrm{a}$ ), but not nontarget Ramos cells (Figure $4 \mathrm{~b}$ ). The dissociation constant of DAA binding to CEM cells $\left(K_{\mathrm{d}}=5.98 \pm 0.78 \mathrm{nM}\right.$, Supplementary Figure S6A) was only slightly higher than that of sgc8 alone $\left(K_{\mathrm{d}}=0.8 \mathrm{~nm}\right),{ }^{26}$ indicating strong binding affinity. The slightly weaker binding affinity of DAA than that of $\mathrm{sgc} 8$ presumably results from conformational changes caused by drug conjugation. DAAs formed from aptamers with commonly used daunorubicin or enhanced permeability and retention also specifically recognized target cells with strong binding affinities (Supplementary Figure S6B and D), as did DAAs formed from aptamers KK1B10 and TD05 and modified aptamer sgc8-(CGA) 10 (Supplementary Figure S6E and G). This indicates that $3 \mathrm{D}$ structural folding was maintained in DAAs, providing the basis for DAA-based active targeted drug delivery.

Using the sgc8-Dox adduct as a model, the intracellular behaviors of free drugs and drugs delivered via DAAs were examined by confocal laser scanning microscopy. Target CEM cells were incubated with free Dox and sgc8-Dox adduct, respectively, followed by transferrinAlexa633 staining to localize endosomes prior to microscopic observation at different time points. Free Dox rapidly entered the cells and ac cumulated in the nucleus (Supplementary Figure S7A). Cells treated with DAAs also displayed a gradual drug uptake and release from DAAs, as well as subsequent accumulation in the nucleus
(Supplementary Figure S7B). Compared with free drugs, the corresponding weaker drug fluorescence in DAA-treated cells was most likely due to: (1) slower uptake of DAAs compared with free drugs and (2) quenched drug fluorescence in DAAs until release. Furthermore, confocal laser scanning microscopy (Figure 4c) identified Dox localized in cytoplasm during the early stages of cell permeation, followed by gradual accumulation in the nucleus, while the aptamer remained in the cytoplasm. Dox release from DAAs and accumulation in the nucleus provide the basis for optimal inhibition of cancer cell proliferation.

The cytotoxicity of sgc8-Dox adduct was evaluated by using an 3-(4,5-dimethylthiazol-2-yl)-5-(3-carboxymethoxyphenyl)-2-(4-sulfophenyl)-2H-tetrazolium (MTS) assay. As a control, free Dox showed dose-dependent cytotoxicity in both CEM cells and Ramos cells (Figures $4 \mathrm{~d}$ and $\mathrm{e}$ ). In contrast, cytotoxicity of sgc8-Dox adduct comparable to that of free Dox was observed only in target CEM cells, indicating potent and selective cytotoxicity of sgc8-Dox adduct. As a control, aptamer (without drugs) with the same treatment and purification failed to induce a significant cytotoxicity (Supplementary Figure S8), verifying the biocompatibility of aptamers and removing the concern about toxicity caused by residual formaldehyde in DDAs.

\section{In vivo evaluation of DAAs for targeted drug delivery}

For optimal evaluation of DAAs for drug delivery in a complicated in vivo environment, another adduct prepared from a control DNA $\left((\mathrm{CGA})_{10}\right)$ and Dox was also used. Flow cytometry results verified that neither this control DNA nor the corresponding adducts were able to specifically recognize CEM cells (Supplementary Figure S9). A tumor xenograft mouse model was established by subcutaneously implanting CEM cells into NOD. Cg-Prikdc (scid) IL2 mice to evaluate DAAs 
a

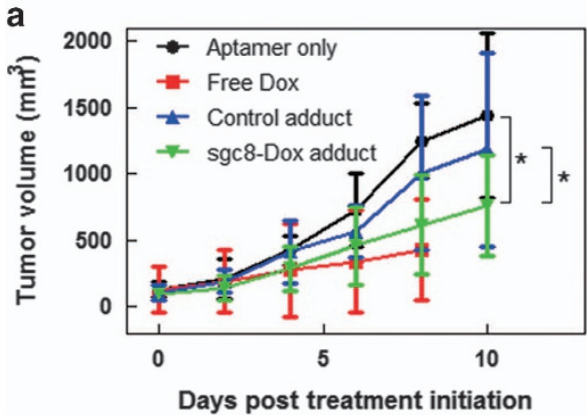

C

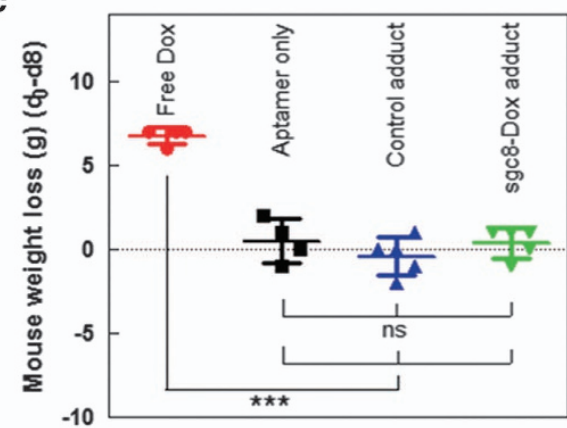

b

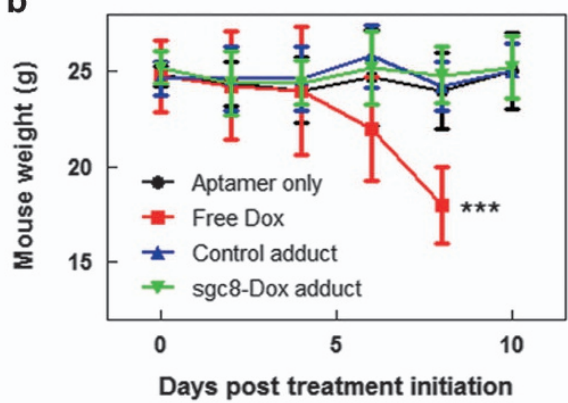

d

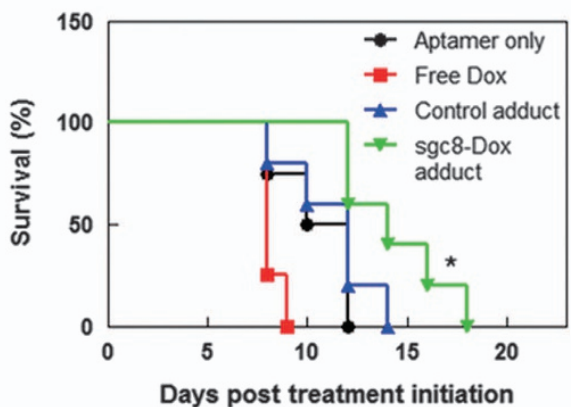

Figure 5 Potent antitumor efficacy and reduced side effects of drug-DNA adducts for targeted drug delivery in a tumor xenograft mouse model. CEM xenograft mouse tumor model was developed by s.c. injection of CEM cells in the backs of NOD.Cg-Prkdc (scid) IL2 mice. Mice were divided into four groups that were treated by i.v. injections with ( $I$ ) sgc8, (ii) free doxorubicin, (iii) control adduct and (iv) sgc8-doxorubicin adduct, respectively. (a) Mouse tumor volume up to day 10 after treatment initiation (mean \pm s.d.; $n=5$ ). Asterisks on day 10 represent significant differences between tumor volumes of mice in $i)$ and $i$ ) or between iii) and iv) ( ${ }^{*} P<0.05$; Student's $t$-test). (b and $\left.\mathbf{c}\right)$ Mouse weight (b) up to day 10 after treatment initiation and mouse weight loss at day 8 (c) (mean \pm s.d.; $n=5$ ). Asterisks represent significant differences between mouse weight (loss) in $i$ ) and other groups (*** $P<0.001$; one-way analysis of variance with Newman-Keuls post hoc test). (d) Survival percentage of mice after treatment initiation $\left({ }^{*} P<0.05\right.$; Log-rank (Mantel-Cox) test).

in vivo for targeted drug delivery. After tumor initiation, mice were divided into four groups for comparative efficacy study, in which the following regimens were respectively administered by i.v. injections every other day: (i) aptamer (sgc8), (ii) free Dox, (iii) control DNADox adduct and (iv) sgc8-Dox adduct. The Dox dosage was kept the same in groups ii), iii) and iv) at $3 \mathrm{mg} \mathrm{kg}^{-1}$, a high dosage for this mouse species. The DNA dosage in groups (i) and (iv) was the same. Tumor sizes and mouse weight (as an indicator of side effects) were measured every other day. The mice treated with free Dox or sgc8-Dox adduct had significantly lower tumor growth rates than those treated with aptamers alone or control adduct (Figure 5a, Supplementary Figure S10). This demonstrates the potent antitumor efficacy of sgc8-Dox adduct. Compared with control adduct, sgc8-Dox adduct induced significant inhibition of tumor growth, demonstrating the selectivity of DAA-mediated drug delivery. The partial tumor growth inhibition induced by control adduct presumably resulted from gradual Dox release from the adduct at a physiological temperature before blood clearance.

One primary goal of targeted drug delivery is to reduce nonspecific drug toxicity in normal tissues and the resultant side effects. In this study, although free Dox induced dramatic inhibition of tumor growth, it caused severe side effects at this dosage, as indicated by the rapid weight loss (average $27.3 \%$ on day 8 ; Figures $5 \mathrm{~b}$ and $\mathrm{c}$ ) and morbidity (Supplementary Figure S10), which eventually caused early mortality. In contrast, mice treated with all other regimens had significantly lower to negligible weight loss. While these results indicate that DAA-mediated targeted drug delivery dramatically reduced the side effects of Dox, they also demonstrate that DAAs may exhibit higher maximal tolerated dosage than the free drug counterparts. This is particularly significant for cancer chemotherapy, given that dosage escalation is needed for many chemotherapeutics to achieve an optimal therapeutic efficacy. Consistently, DAA-treated mice had a higher survival rate than other groups of mice. In contrast, mice treated with free Dox showed the lowest average survival rate as a consequence of severe side effects (Figure 5d).

Tissue was collected from the treated mice for histopathological and immunohistochemical analyses. Specifically, in hematoxylin and eosin histopathological analysis, significant tumor necrosis was observed only in mice treated with free Dox or DAAs (Figure 6a), and apparent heart tissue deformation was observed only in mice treated with free Dox (Figure 6b). No significant change was shown in the kidney (Supplementary Figure S11). In situ immunohistochemical analysis of cleaved caspase- 3 in tumors and hearts was used to elucidate apoptosis, by using a cleaved caspase- 3 (Asp175) antibody ${ }^{38}$. Tumors from mice treated with free Dox or DAAs displayed significant caspase-3 cleavage (Figure 6c). Mild caspase-3 cleavage was also observed in mice treated with aptamer alone or control adduct, presumably resulting from immune response or drug escaped from the adduct. Remarkably, no detectable cleaved caspase- 3 in heart was shown in mice treated with either aptamer alone, control adduct or DAAs, in contrast to a mild cleaved caspase- 3 in mice treated with free Dox (Figure 6d). The amelioration of tissue deformation and apoptosis in the heart indicates DAAs as a potential new therapeutic approach for the prevention of cardiomyopathy, the most dangerous side effect caused by cardiotoxicity of anthracycline drugs and leading to lethal congestive heart failure. Taken together, these data clearly suggest that DAAs have potent antitumor efficacy and can reduce side effects, thereby achieving targeted cancer therapy. 

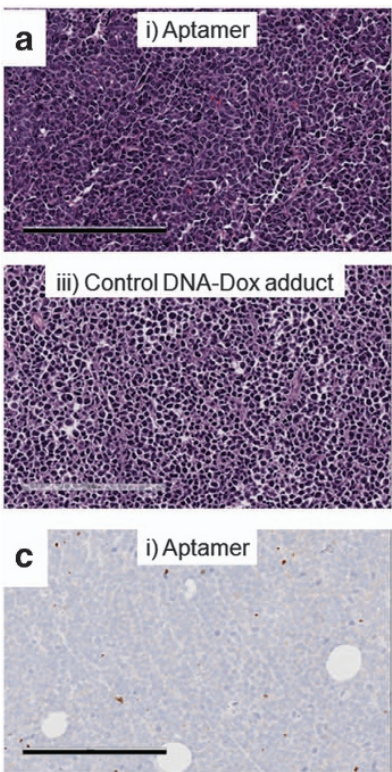

iii) Control DNA-Dox adduct

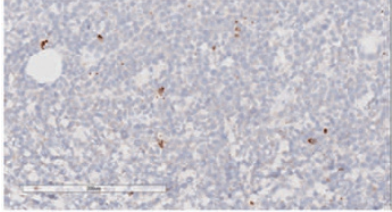

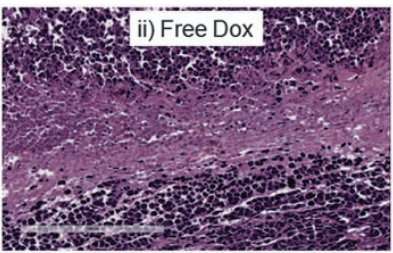

iv) Aptamer-Dox adduct
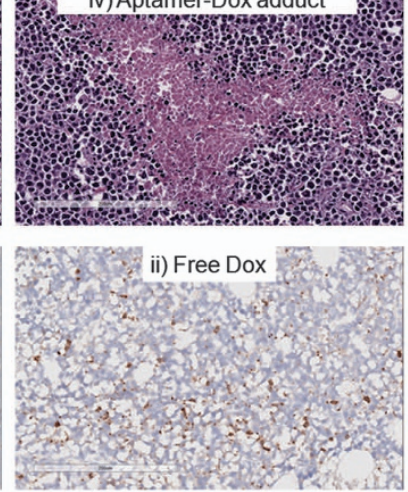

iv)Aptamer-Dox adduct

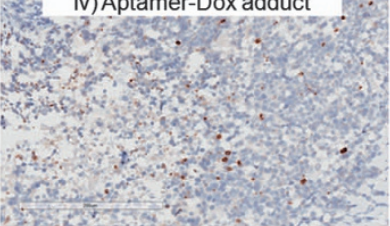

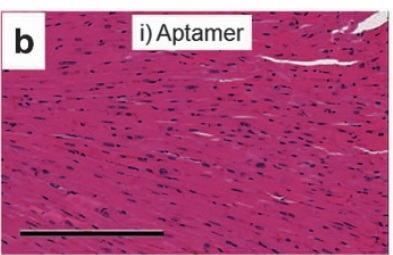
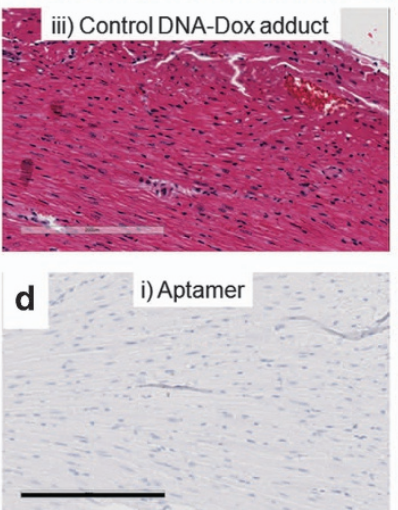

iii) Control DNA-Dox adduct

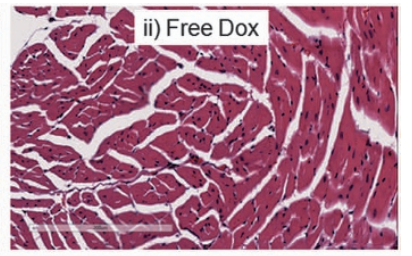

iv) Aptamer-Dox adduct
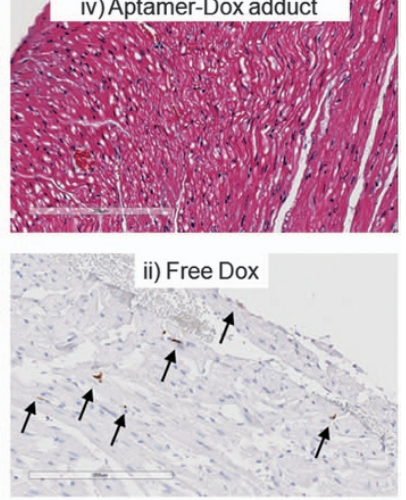

iv)Aptamer-Dox adduct
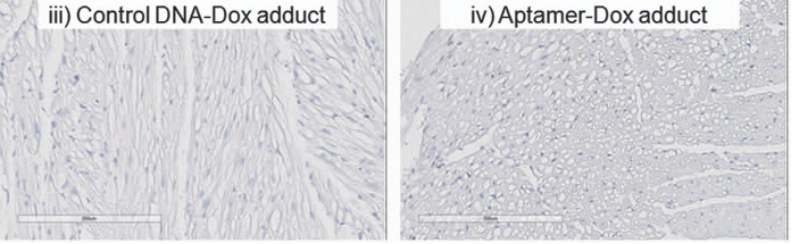

Figure 6 Representative images of tissue analyses verifying drug-aptamer adducts-induced potent antitumor efficacy and reduction of side effects. (a and b) Hematoxylin and eosin histopathological analysis of tumors (a) and hearts (b) indicating tumor necrosis in mice treated with free doxorubicin or drug-aptamer adducts, as well as reduction of heart tissue deformation in mice treated with drug-aptamer adducts, compared with free doxorubicin. (c and d) In situ immunohistochemical analysis of cleaved Caspase-3 (brown) in tumors (c) and hearts (d) indicating tumor apoptosis in mice treated with free doxorubicin or drug-aptamer adducts, but no detectable apoptosis in hearts from mice treated with drug-aptamer adducts, compared with free doxorubicin (arrows indicate apoptosis). Blue: cell nuclei stained by hematoxylin. (scale bar: $200 \mu \mathrm{m}$ ).

\section{CONCLUSIONS}

Targeted drug delivery is promising to overcome complications in conventional cancer chemotherapy. DNA, serving as molecular recognition elements or structural building blocks of drug nanocarriers, has been extensively studied for targeted drug delivery. Inspired by natural adduct formation between many carcinogens and genomic DNA, here we developed a simple, yet versatile and programmable platform of synthetic DDAs as site-specific drug-DNA conjugates for a targeted drug delivery. With a simple reaction, DDA formation made use of inherent reactive groups on DNA, thus avoiding prior chemical modification of DNA or any complicated organic synthesis. Anthracycline drugs were specifically conjugated on deoxyguanosine of DNA, making it programmable to design DNA and drugs for DDA-based drug delivery systems. Due to deoxyguanosine-specific drug conjugation, one DNA can typically be conjugated with multiple copies of drugs, resulting in a high drug payload capacity. DDAs were resistant to nuclease degradation, providing a novel strategy to combat nuclease degradation for DNA-based drug carriers. DDAs were exceptionally stable at a relatively low temperature, yet drugs were gradually released at a physiological temperature. Mediated by hybridization, drug-DNA nanoadducts were constructed by using DDA building blocks, which makes this platform promising for nanocarrier-based targeted drug delivery. The aptamer backbones of DAAs maintained selectivity in cancer cell recognition and drug delivery, resulting in selective cytotoxicity in target cells. In a xenograft tumor mouse model, DAAs showed potent and specific antitumor efficacy with significantly reduced side effects compared with free drugs. Remarkably, histopathological and immunohistochemical analyses of tumors and hearts verified that the Dox-aptamer adduct induced potent toxicity in the tumor and dramatically ameliorated tissue deformation and apoptosis in the heart. This provides a new therapeutic approach to prevent cardiomyopathy, the most dangerous side effect caused by anthracycline drugs and leading to lethal congestive heart failure $(50 \%$ mortality). In another independent study, we have validated AS1411-Dox adduct for targeted liver cancer therapy, which will be reported elsewhere. Overall, synthetic DDAs provide a simple and programmable platform of site-specific drug-DNA conjugation, which is promising for scale-up production and broad application in DNAbased targeted drug delivery.

\section{CONFLICT OF INTEREST}

The authors declare no conflict of interest.

\section{ACKNOWLEDGEMENTS}

The authors thank Dr Kathryn R. Williams for critical manuscript review. We acknowledge the Interdisciplinary Center for Biotechnology Research (ICBR) at the University of Florida for help with confocal microscopy analysis, Novatia for ESI-MS analysis, Dr Mei Zhang for assistance with mouse work, and Molecular Pathology Core at the University of Florida for assistance with tissue analysis. This work was supported by the National Institutes of Health (GM079359, and CA133086), and by National Key Scientific Program of China (2011CB911000), NSFC (21221003, 21327009), National Instrumentation Program (2011YQ030124). 
1 Minotti, G., Menna, P., Salvatorelli, E., Cairo, G. \& Gianni, L. Anthracyclines: molecular advances and pharmacologic developments in antitumor activity and cardiotoxicity. Pharmacol. Rev. 56, 185-229 (2004).

2 Chatterjee, K., Zhang, J., Honbo, N. \& Karliner, J. S. Doxorubicin cardiomyopathy. Cardiology 115, 155-162 (2010).

3 Peer, D., Karp, J. M., Hong, S., Farokhzad, O. C., Margalit, R. \& Langer, R. Nanocarriers as an emerging platform for cancer therapy. Nat. Nanotechnol. 2, 751-760 (2007).

$4 \mathrm{Li}, \mathrm{X}$., Fang, T. \& Boons, G.-J. Preparation of well-defined antibody-drug conjugates through glycan remodeling and strain-promoted azide-alkyne cycloadditions. Angew. Chem. Int. Ed. Engl. 126, 7307-7310 (2014).

5 Santra, S., Kaittanis, C., Santiesteban, O. J. \& Perez, J. M. Cell-specific, activatable, and theranostic prodrug for dual-targeted cancer imaging and therapy. J. Am. Chem. Soc. 133, 16680-16688 (2011).

6 Allen, T. M. Ligand-targeted therapeutics in anticancer therapy. Nat. Rev. Cancer 2, 750-763 (2002)

7 Cao, Z., Tong, R., Mishra, A., Xu, W., Wong, G. C. L., Cheng, J. \& Lu, Y. Reversible cellspecific drug delivery with aptamer-functionalized liposomes. Angew. Chem. Int. Ed. Engl. 48, 6494-6498 (2009).

8 Wang, F., Wang, Y.-C., Dou, S., Xiong, M.-H., Sun, T.-M. \& Wang, J. Doxorubicintethered responsive gold nanoparticles facilitate intracellular drug delivery for overcoming multidrug resistance in cancer cells. ACS Nano 5, 3679-3692 (2011).

9 Lo, P. K., Karam, P., Aldaye, F. A., McLaughlin, C. K., Hamblin, G. D., Cosa, G. \& Sleiman, H. F. Loading and selective release of cargo in DNA nanotubes with longitudinal variation. Nat. Chem. 2, 319-328 (2010).

10 Jiang, Q., Song, C., Nangreave, J., Liu, X., Lin, L., Qiu, D., Wang, Z.-G., Zou, G., Liang, X., Yan, H. \& Ding, B. DNA origami as a carrier for circumvention of drug resistance. J. Am. Chem. Soc. 134, 13396-13403 (2012).

11 Chang, M., Yang, C.-S. \& Huang, D.-M. Aptamer-conjugated dna icosahedral nanoparticles as a carrier of doxorubicin for cancer therapy. ACS Nano $\mathbf{5}$, 6156-6163 (2011).

12 Lee, H., Lytton-Jean, A. K. R., Chen, Y., Love, K. T., Park, A. I., Karagiannis, E. D., Sehgal, A., Querbes, W., Zurenko, C. S., Jayaraman, M., Peng, C. G., Charisse, K., Borodovsky, A., Manoharan, M., Donahoe, J. S., Truelove, J., Nahrendorf, M., Langer, R. \& Anderson, D. G. Molecularly self-assembled nucleic acid nanoparticles for targeted in vivo siRNA delivery. Nat. Nanotechnol. 7, 389-393 (2012).

13 Zhu, G., Zheng, J., Song, E., Donovan, M., Zhang, K., Liu, C. \& Tan, W. Self-assembled, aptamer-tethered DNA nanotrains for targeted transport of molecular drugs in cancer theranostics. Proc. Natl Acad. Sci. USA 110, 7998-8003 (2013).

14 Zhu, G., Hu, R., Zhao, Z., Chen, Z., Zhang, X. \& Tan, W. Noncanonical self-assembly of multifunctional DNA nanoflowers for biomedical applications. J. Am. Chem. Soc. 135 16438-16445 (2013)

15 Zhu, G., Meng, L., Ye, M., Yang, L., Sefah, K., O'Donoghue, M. B., Chen, Y, Xiong, X., Huang, J., Song, E. \& Tan, W. Self-assembled aptamer-based drug carriers for bispecific cytotoxicity to cancer cells. Chem. Asian J. 7, 1630-1636 (2012).

16 Wu, C., Han, D., Chen, T., Peng, L., Zhu, G., You, M., Qiu, L., Sefah, K., Zhang, X. \& Tan, W. Building a multifunctional aptamer-based dna nanoassembly for targeted cancer therapy. J. Am. Chem. Soc. 135, 18644-18650 (2013).

17 Wang, K., You, M., Chen, Y., Han, D., Zhu, Z., Huang, J., Williams, K., Yang, C. J. \& Tan, W. Self-assembly of a bifunctional dna carrier for drug delivery. Angew. Chem. Int. Ed. Engl. 50, 6098-6101 (2011).

18 Bagalkot, V., Farokhzad, O. C., Langer, R. \& Jon, S. An aptamer-doxorubicin physical conjugate as a novel targeted drug-delivery platform. Angew. Chem. Int. Ed. Engl. 45, 8149-8152 (2006).

19 Meng, L., Yang, L., Zhao, X., Zhang, L., Zhu, H., Liu, C. \& Tan, W. Targeted delivery of chemotherapy agents using a liver cancer-specific aptamer. Plos ONE 7, e33434 (2012).

20 Kortylewski, M., Swiderski, P., Herrmann, A., Wang, L., Kowolik, C., Kujawski, M., Lee, H., Scuto, A., Liu, Y., Yang, C., Deng, J., Soifer, H. S., Raubitschek, A., Forman, S., Rossi, J. J., Pardoll, D. M., Jove, R. \& Yu, H. In vivo delivery of siRNA to immune cells by conjugation to a TLR9 agonist enhances antitumor immune responses. Nat. Biotechnol. 27, 925-932 (2009).

21 Weiner, G. J., Liu, H.-M., Wooldridge, J. E., Dahle, C. E. \& Krieg, A. M. Immunostimulatory oligodeoxynucleotides containing the $\mathrm{CpG}$ motif are effective as immune adjuvants in tumor antigen immunization. Proc. Natl Acad. Sci. USA 94, 10833-10837 (1997).
22 Ellington, A. D. \& Szostak, J. W. In vitro selection of RNA molecules that bind specific ligands. Nature 346, 818-822 (1990)

23 Tuerk, C. \& Gold, L. Systematic evolution of ligands by exponential enrichment: RNA ligands to bacteriophage T4 DNA polymerase. Science 249, 505-510 (1990).

24 Keefe, A. D., Pai, S. \& Ellington, A. Aptamers as therapeutics. Nat. Rev. Drug Discov. 9, 537-550 (2010).

25 Zhu, G., Ye, M., Donovan, M. J., Song, E., Zhao, Z. \& Tan, W. Nucleic acid aptamers: an emerging frontier in cancer therapy. Chem. Commun. 48, 10472-10480 (2012).

26 Shangguan, D., Li, Y., Tang, Z., Cao, Z., Chen, H. W., Mallikaratchy, P., Sefah, K., Yang, C. J. \& Tan, W. Aptamers evolved from live cells as effective molecular probes for cancer study. Proc. Natl Acad. Sci. USA 103, 11838-11843 (2006).

27 Sefah, K., Tang, Z., Shangguan, D., Chen, H., Lopez-Colon, D., Li, Y., Parekh, P., Martin, J., Meng, L., Phillips, J. A., Kim, Y. \& Tan, W. Molecular recognition of acute myeloid leukemia using aptamers. Leukemia 23, 235-244 (2009).

28 Tang, Z., Shangguan, D., Wang, K., Shi, H., Sefah, K., Mallikaratchy, P., Chen, H. W., $\mathrm{Li}, \mathrm{Y}$. \& Tan, W. Selection of aptamers for molecular recognition and characterization of cancer cells. Anal. Chem. 79, 4900-4907 (2007)

29 Alama, A., Barbieri, F., Cagnoli, M. \& Schettini, G. Antisense oligonucleotides as therapeutic agents. Pharmacol. Res. 36, 171-178 (1997).

30 Huang, Y.-F., Shangguan, D., Liu, H., Phillips, J. A., Zhang, X., Chen, Y. \& Tan, W. Molecular assembly of an aptamer-drug conjugate for targeted drug delivery to tumor cells. ChemBioChem 10, 862-868 (2009).

31 Wang, R., Zhu, G., Mei, L., Xie, Y., Ma, H., Ye, M., Qing, F.-L. \& Tan, W. Automated modular synthesis of aptamer-drug conjugates for targeted drug delivery. J. Am. Chem. Soc. 136, 2731-2734 (2014).

32 Wilson, C. \& Keefe, A. D. Building oligonucleotide therapeutics using non-natural chemistries. Curr. Opin. Chem. Biol. 10, 607-614 (2006).

33 Rosi, N. L., Giljohann, D. A., Thaxton, C. S., Lytton-Jean, A. K. R., Han, M. S. \& Mirkin, C. A. Oligonucleotide-modified gold nanoparticles for intracellular gene regulation. Science 312, 1027-1030 (2006).

34 van Rosmalen, A., Cullinane, C., Cutts, S. M. \& Phillips, D. R. Stability of adriamycin-induced DNA adducts and interstrand crosslinks. Nucleic Acids Res. 23, 42-50 (1995).

35 Cutts, S. M. \& Phillips, D. R. Use of oligonucletides to define the site of interstand cross-links induced by Adriamycin. Nucleic Acids Res. 23, 2450-2456 (1995).

36 Zeman, S. M., Phillips, D. R. \& Crothers, D. M. Characterization of covalent AdriamycinDNA adducts. Proc. Natl Acad. Sci. USA 95, 11561-11565 (1998).

37 Swift, L. P., Rephaeli, A., Nudelman, A., Phillips, D. R. \& Cutts, S. M. Doxorubicin-DNA adducts induce a non-topoisomerase II-mediated form of cell death. Cancer Res. 66, 4863-4871 (2006).

38 Nicholson, D. W., Ali, A., Thornberry, N. A., Vaillancourt, J. P., Ding, C. K., Gallant, M., Gareau, Y., Griffin, P. R., Labelle, M., Lazebnik, Y. A., Munday, N. A., Raju, S. M., Smulson, M. E., Yamin, T.-T., Yu, V. L. \& Miller, D. K. Identification and inhibition of the ICE/CED-3 protease necessary for mammalian apoptosis. Nature 376, 37-43 (1995).

39 Shangguan, D., Cao, Z., Meng, L., Mallikaratchy, P., Sefah, K., Wang, H., Li, Y. \& Tan, W. Cell-specific aptamer probes for membrane protein elucidation in cancer cells. J. Proteo. Res 7, 2133-2139 (2008).

40 Heinlein, T., Knemeyer, J.-P., Piestert, O. \& Sauer, M. Photoinduced electron transfer between fluorescent dyes and guanosine residues in DNA-hairpins. J. Phy. Chem. B 107, 7957-7964 (2003).

(c) (1) (2) This work is licensed under a Creative Commons Attribution-NonCommercial-ShareAlike 4.0 International License. The images or other third party material in this article are included in the article's Creative Commons license, unless indicated otherwise in the credit line; if the material is not included under the Creative Commons license, users will need to obtain permission from the license holder to reproduce the material. To view a copy of this license, visit http://creativecommons.org/licenses/ by-nc-sa/4.0/

Supplementary Information accompanies the paper on the NPG Asia Materials website (http://www.nature.com/am) 